\title{
Procedimento didático para a modelagem computacional da amêndoa do cupuaçu em coordenadas cartesianas
}

Jorge Henrique de Oliveira Sales jhosales@uesc.br

0000-0003-1992-3748

Universidade Estadual de Santa Cruz, Ilhéus, Bahia, Brasil.

Geovana Pires Araujo Lima geovanapires1907@hotmail.com 0000-0003-3062-4837

Universidade Estadual de Santa Cruz, Ilhéus, Bahia, Brasil.

\begin{abstract}
RESUMO
Este artigo propõe um exemplo de didático voltado para o público da graduação e pósgraduação para a modelagem de materiais utilizando um sistema de coordenadas cartesianas. O material investigado foi a amêndoa do cupuaçu, com objetivo de descrever a curva de resfriamento da amêndoa. Para isso, a metodologia empregada consistiu na resolução analítica da equação de Fourier em coordenadas cartesianas e simulação computacional do modelo. A simulação avaliou a dissipação de calor da amêndoa, que apresentava temperatura inicial de $70^{\circ} \mathrm{C}$, e foi submetida à temperatura ambiente de $25^{\circ} \mathrm{C}$. A curva de resfriamento da amêndoa do cupuaçu revela que uma rápida redução na temperatura nos primeiros minutos de simulação, assumindo um comportamento mais lento a partir dos 30 minutos.
\end{abstract}

PALAVRAS-CHAVE: Transferência de calor. Equação de Fick. Ensino. 


\section{INTRODUÇÃO}

A utilização de modelos computacionais no contexto educacional é fundamental para que os discentes compreendam sua aplicação, e possam representar sistemas reais por meio de modelos computacionais e avaliar o seu comportamento sem a necessidade da construção de um sistema real, evitando elevados custos (CHWIF; MEDINA, 2014; COMSOL, 2017).

Modelar é representar um Sistema Físico Real, ou parte dele, em forma simbólica, convenientemente preparada para predizer ou descrever o comportamento em qualquer tempo futuro. Os modelos podem ser classificados basicamente em quatro tipos, a saber: Icônico, Diagramático, Matemático e computacional.

Modelo icônico é aquele que representa, de forma mais fiel possível do sistema físico real. Podem ser bi ou tridimensionais, confeccionados em tamanho natural (escala 1x1) ou em escala ampliada ou reduzida, exemplo são os modelos em miniatura de carro ou prédios.

Modelo diagramático é um conjunto de linhas e símbolos que representam a estrutura ou o comportamento do sistema físico real. Um exemplo são os esquemas de placas de circuitos eletrônicos.

Modelos matemáticos é uma idealização, onde são usadas técnicas de construção lógica, não necessariamente naturais e, certamente, não completas. Exemplos são os modelos matemáticos para escoamento, modelos para partículas elementares...

Os modelos computacionais usam programas e podem ser aplicados em diversos sistemas, no caso deste trabalho, será a amêndoa do cupuaçu, cuja motivação vem da abundância do fruto, figura 1, na região em que a Universidade (Universidade Estadual da Santa Cruz - UESC), onde foram realizadas as pesquisas, está inserida.

O cupuaçu é um fruto nativo Figura 1, com valor econômico, usado aqui como modelo. O cupuaçu é composto por $45,20 \%$ de casca, $37,30 \%$ de polpa, $15 \%$ de semente e $2,5 \%$ de placenta (SOUZA et al., 1999), entretanto, praticamente só a polpa é aproveitada, sendo usada na fabricação de polpas, cosméticos, doces entre outros (YANG et al., 2003). A amêndoa do cupuaçu é praticamente descartada no processo de beneficiamento, porém ela apresenta excelentes características nutritivas e propriedades muito próximas as da amêndoa do cacau, podendo ser usado na fabricação de produtos como chocolates e derivados (COHEN et al., 2003). 


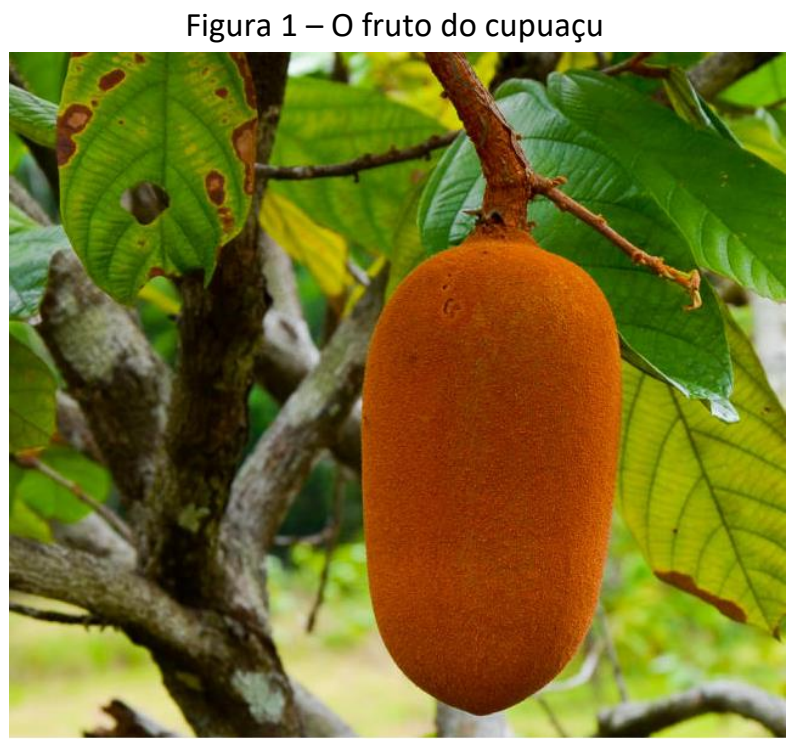

Fonte: Embrapa (2016).

O "chocolate" de cupuaçu (cupulate) começou a ser pesquisado pelo CPATU (Centro de Pesquisa Agro florestal da Amazônia Oriental) da Embrapa, em 1986, o processamento é o mesmo para a fabricação do chocolate com o cacau e apresenta algumas vantagens, como menor custo e a fermentação do cupuaçu a qual é favorecida pelos resíduos orgânicos da polpa que ficam colados na semente (NAZARÉ et al., 1990). Entretanto, a literatura não apresenta resultados de pesquisas quanto à curva de resfriamento da amêndoa do cupuaçu (SALES; CÂNDIDA, 2016; LIMA; SALES, 2017).

Conhecer o comportamento térmico de materiais biológicos é fundamental para a engenharia, com isso é possível prognosticar a taxa de secagem, velocidade da propagação do calor e a distribuição de calor em materiais biológicos de composição e formas variadas (SASSERON, 1984). Além disso, possibilita o desenvolvimento de produtos tecnológicos que agreguem maior valor à secagem e armazenamento dos grãos, pois permitem que o beneficiamento ocorra de maneira adequada levando em consideração as características peculiares de cada tipo de produto agrícola (LIMA, 2018).

Desta forma, este estudo apresenta a solução da equação da difusão do calor, ou equação de Fourier, e obtendo a curva de resfriamento na geometria do tipo paralelepípedo, aproximando assim do formato de uma amêndoa do cupuaçu. A modelagem computacional se dar com dissipação do calor para o ambiente, sendo que amêndoa inicialmente apresenta $70^{\circ} \mathrm{C}$ e dissipa para o ambiente, possuindo uma temperatura $25^{\circ} \mathrm{C}$.

\section{MATERIAIS E MÉTODOS}

A metodologia empregada no presente trabalho consistiu na resolução analítica da Equação de Fourier (SALES; LIMA, 2018) para o calor e a simulação computacional da curva de resfriamento da amêndoa do cupuaçu. 
O método é analítico para solucionar a equação de Fick (SALES; LIMA, 2018) da difusão de massa $D$ em coordenadas cartesianas, para amêndoa do cupuaçu. No caso tridimensional em coordenadas cartesianas é dada pela equação

$$
\frac{\partial \mathrm{M}(x, y, z, t)}{\partial t}=\frac{\partial}{\partial x}\left(\mathrm{D} \frac{\partial \mathrm{M}(x, y, z, t)}{\partial x}\right)+\frac{\partial}{\partial y}\left(\mathrm{D} \frac{\partial \mathrm{M}(x, y, z, t)}{\partial y}\right)+\frac{\partial}{\partial z}\left(\mathrm{D} \frac{\partial \mathrm{M}(x, y, z, t)}{\partial z}\right)
$$

onde $M$ é a massa ou o número de átomos, que está se difundindo perpendicularmente através de uma área reta $\mathrm{A}$ em um tempo t. A equação correspondente a esta para condução de calor é a lei de Fourier, que é obtida substituindo $\mathrm{M}(x, y, z, t)$ por $\Psi$ (temperatura) e $\mathrm{D}$ por $\alpha$ (difusão térmica), assumindo-se que as propriedades térmicas, como calor específico e densidade de massa, sejam constantes, caso contrário não poderíamos usar a técnica de separação de variáveis para resolver a Eq. (1) analiticamente (LIMA, 2018).

A equação usada para a resolução deste problema é a de Fourier que é uma equação diferencial parcial de segunda ordem que descreve a variação de temperatura em um volume constante, onde só ocorre troca de calor por meio da condução térmica e o regime é não estacionário (temperatura varia com o tempo). Essa equação é dada por:

$$
\frac{\partial \Psi(r, t)}{\partial t}=\alpha \nabla^{2} \Psi(r, t)(2)
$$

sendo $\alpha$ é a difusividade térmica e $\Psi(r, t)$ é a temperatura.

A posição de um ponto dentro da amêndoa é $r$ que depende do sistema de coordenada escolhida e o tempo de medida é $t$. $O$ operador $\nabla^{2}$ está associado ao ponto do espaço.

A primeira técnica usada na solução na Eq. (2) é a separação de variáveis (ARFKEN; WERBER, 2007) entre a variável espacial $r$ e temporal $t$ :

$$
\Psi(r, t)=\psi(r) T(t)(\mathbf{3})
$$

onde $\psi(r)$ é função só do espaço e $T(t)$ função apenas do tempo. Substituindo (3) em (2), tem se

$$
\frac{1}{\alpha \mathrm{T}} \frac{\mathrm{dT}}{\mathrm{d} t}=\frac{\nabla^{2} \psi(r)}{\psi(r)}
$$

Assim, é separado da temperatura $\Psi(r, t)$ a parte espacial $\psi(r)$ da temporal $\mathrm{T}(t)$. Dessa forma, o modelo geométrico se concentra na parte espacial isolado da temporal. Como o primeiro membro é função somente de t e no segundo, uma função de pontos espaciais, então a igualdade só ocorrerá se for constante real ou complexa, cuja escolha dependerá das condições de contorno do problema. No caso deste trabalho é complexo por causa das funções espaciais serem periódicas, então:

$$
\frac{1}{\alpha T} \frac{d T}{d t}=\frac{\nabla^{2} \psi(r)}{\psi(r)}=-\lambda^{2}
$$

A solução temporal da Eq. (4) vem da equação diferencial de primeira ordem, ou seja:

$$
\frac{1}{\alpha T} \frac{d T}{d t}=-\lambda^{2}
$$


A constante $\lambda^{2}$ depende das condições de contorno do sistema (amêndoa) para solução particular de (5). A solução geral é dada por:

$$
\frac{1}{\alpha T(t)} \frac{d T(t)}{d t}=-\lambda^{2} \rightarrow \frac{d T(t)}{T(t)}=-\alpha \lambda^{2} d t \rightarrow \int \frac{d T(t)}{T(t)}=-\alpha \lambda^{2} \int d t
$$

resultando

$$
T(t)=G e^{-\alpha \lambda^{2} t}
$$

onde $\mathrm{G}$ é uma constante que depende das condições iniciais do sistema. Essas condições serão apresentadas na seção de resultados.

A parte espacial da equação (4) é,

$$
\frac{\nabla^{2} \psi(r)}{\psi(r)}=-\lambda^{2}
$$

Em muitos problemas físicos, as condições de contorno são tais que os valores da função (ou de sua derivada) são especificados em curvas ou superfícies. A escolha de um particular sistema de coordenadas deve ser motivada pela forma geométrica do corpo em estudo e resulta numa melhor adequação e validade dos resultados obtidos (SALES; LIMA, 2018).

Tendo em vista a complexidade da solução da Eq. (1) no problema proposto, e visando simplificar o método, algumas considerações foram adotadas: a) $O$ sólido é homogêneo e com propriedades físicas constantes; b) $\mathrm{O}$ campo do teor da umidade é assimétrico em torno do eixo $z$ ao longo do processo; c) A secagem ocorre em condições de equilíbrio na superfície, com teor de umidade constante, igual ao teor de umidade de equilíbrio; d) $O$ encolhimento do material é desprezível durante o processo de secagem; e) O campo do teor de umidade é uniforme no início da secagem; f) $O$ único mecanismo de transporte de umidade no interior do sólido é o de difusão em fase líquida; g) O coeficiente de difusão é constante; h) Temperatura na superfície da amêndoa é constante a $0{ }^{\circ} \mathrm{C}$, isso facilita os cálculos nas condições de contorno.

Sendo que para cada sistema de coordenada geométrica o operador laplaciano $\nabla^{2} \psi$ tem uma forma diferente, no caso das coordenadas cartesianas, o operador laplaciano consiste em

$$
\nabla^{2} \psi=\frac{\partial^{2} \psi}{\partial x^{2}}+\frac{\partial^{2} \psi}{\partial y^{2}}+\frac{\partial^{2} \psi}{\partial z^{2}}
$$

Neste artigo é usado o sistema de coordenada cartesiano ou retangular por causa da sua simplicidade nas soluções da Eq. (1) via separação de variáveis. Trabalho recente (SALES; LIMA, 2018) mostra como mudança do sistema de coordenadas no laplaciano afetas o resultado da curva da temperatura para o cacau. No caso do cupuaçu o mesmo estudo foi realisado por Lima (LIMA, 2018).

Para a simulação computacional, neste trabalho, o software utilizado para a realização da simulação foi o COMSOL Multiphysics 5.1 (LIMA, 2018), os gráficos foram gerados no Origin Pro 8. As especificações do computador e do ferramental utilizado é apresentado na Tabela 1. Mas, os resultados deste artigo podem ser reproduzidos em qualquer outro equipamento ou software com essas especificações que são mínimas, caso contrário o software usado pode não funciona. 
Tabela 1 - Item e especificações do ferramental utilizado

$\begin{array}{cc}\text { Item } & \text { Especificação } \\ \text { Processador } & \text { Intel (R) Core (TM) i3-2310M CPU@ 2.10 GHz } \\ \text { Memória (RAM) } & 2.10 \mathrm{GHz} \\ \text { Sistema Operacional } & 4,00 \mathrm{~GB} \\ \text { Software CAE } & \text { Windows } 7 \text { de } 64 \text { Bits } \\ \text { Software gráfico } & \text { COMSOL Multiphysics } 5.1 \\ \text { Software } & \text { OriginPro 8 }\end{array}$

O COMSOL Multiphysics ${ }^{\circledR}$ possui diferentes módulos criados para simular fenômenos físicos específicos, como transferência de calor, fluidodinâmica, mecânica estrutural, reações químicas e eletromagnetismo. Ao adicionar um módulo ao modelo de simulação, as equações que modelam matematicamente o fenômeno físico (comumente equações diferenciais parciais) são carregadas por padrão, sem que o usuário precise determiná-las manualmente. Porém, caso seja necessário, o usuário pode alterar qualquer equação para adequar o seu modelo ao fenômeno que se pretende simular (LIMA; SALES; PORTUGAL, 2015; SOGLIA, 2019).

No presente estudo, o modelo de simulação escolhido foi o "model wizard", consiste em um módulo inteligente, que facilita na construção do modelo. A dimensão do espaço trabalhado foi em três dimensões. O método físico empregado para a resolução do problema foi a transferência de calor "Heat transfer module", este módulo permite analisar efeitos de aquecimento e resfriamento em diferentes sistemas. Oferece também ferramentas de simulação para estudar mecanismos de transferência de calor - condução, convecção e radiação - geralmente associada com outros métodos físicos. Desta forma, o Heat Transfer Module possibilita o estudo de situações onde há geração, absorção ou transferência de calor ou energia, tendo larga aplicação nas indústrias e nos meios científicos. O tipo de estudo no problema foi o "Time Dependent", ou seja, no qual a dependência temporal não é estacionária, pois o calor dentro da amêndoa varia com o tempo. A Figura 2 apresenta a síntese dos passos citados para a preparação do ambiente de simulação. 


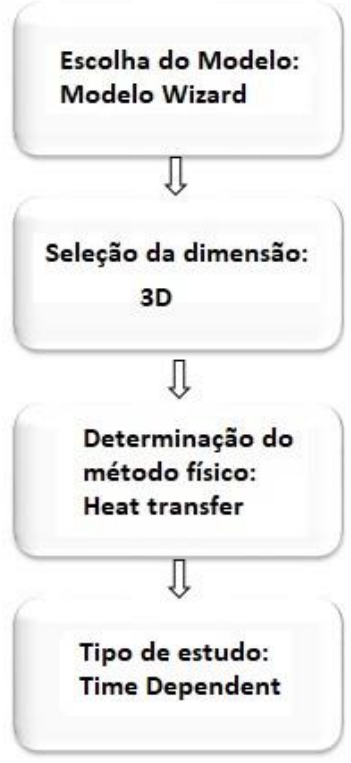

Fonte: LIMA (2018).

Após a preparação do ambiente de simulação, é construída a geometria, para a representação na forma de paralelepípedo usando a malha tetraédrica, com a opção de bloco. O COMSOL possui uma ampla biblioteca de materiais, contendo dados de mais de 2.500 materiais, incluindo elementos, minerais, ligas metálicas, isolantes térmicos, semicondutores e materiais piezelétricos, cada material tem propriedades representadas por funções, para até 24 propriedades (COMSOL, 2017). Entretanto não consta na biblioteca do COMSOL o material que constitui o cupuaçu, desta forma para que a simulação descrevesse o modelo de maneira mais realista possível, foi criado um novo material com as propriedades do cupuaçu.

Após a definição da geometria e do material, é criada a malha de cálculo. A malha escolhida para a simulação do modelo, consistir em uma malha tetraédrica, do tipo fina com construção automática. As ferramentas de criação de malha automáticas e semiautomáticas estão disponíveis no COMSOL Multiphysis ${ }^{\circledR}$, incluindo a criação de malha tetraédrica livre e criação de malha varrida. 0 algoritmo padrão é a criação de malha tetraédrica automática para métodos físicos definidos em sólidos e uma combinação de criação de malha tetraédrica e de camadas limite para fluidos. Pode-se também engendrar uma malha personalizada, definindo a sequência de malhas, esta permite uma combinação de diferentes elementos tetraédricos, prismáticos ou hexaédricos e pode ser parametricamente orientada. O COMSOL suporta também elementos de pirâmides, em malhas importados do SOFTWARE NASTRAN, esta pode ser sucessivamente particionada nos níveis de domínio, contorno e aresta em relação às coordenadas, por meio de operações extras (COMSOL, 2017).

O COMSOL permite separar as diferentes geometrias dos elementos finitos (em 3D: tetraedro, prisma, hexaedro, pirâmide) das "funções de forma dos elementos finitos". Consequentemente oferece grande flexibilidade, e as geometrias suportam funções de primeira, segunda, terceira ordem, sendo que em algumas situações suportam ordens maiores. Na transferência de calor são amplamente usados os elementos finitos de Lagrange (SOGLIA, 2019) também chamado de elementos finitos de base nodal isoparamétrico (COMSOL, 2017). 
Existem diferentes tipos e tamanhos de malhas, quanto mais refinadas mais precisa é a simulação e consequentemente maior o esforço computacional. As malhas de cálculo são essenciais para a realização da simulação, pois a partir destas, as equações diferenciais parciais são solucionadas por meio do método dos elementos finitos, método matemático que se baseia na subdivisão em partes infinitesimais do meio contínuo em elementos que mantém as propriedades de quem os originou. Desta forma os elementos gerados com a discretização são descritos por meio de equações diferenciais e solucionados por modelos matemáticos, fornecendo assim os resultados da simulação (LOTT et al., 2006 apud MCGUINNESS et al., 1992).

Com a malha gerada, o próximo estudo é realizar a simulação. A simulação avaliou o comportamento de resfriamento da amêndoa do cupuaçu, dissipando calor para o ambiente, a temperatura ambiente considerada na simulação foi de $25^{\circ} \mathrm{C}$.

Os resultados da simulação realizada no COMSOL, foram plotados no software Origin Pro 8, que oferece melhor design gráfico, permitindo avaliar melhor o comportamento térmico das amêndoas.

\section{RESULTADOS}

O modelo prismático é constituído por três eixos perpendiculares entre si, estes eixos são representados pelas letras $X, Y$ e Z. Nesta seção a amêndoa do cupuaçu será representada por meio de coordenadas cartesianas, modelo prismático, que é representado na Figura 3.

Figura 3 - Modelo prismático para a amêndoa do cupuaçu
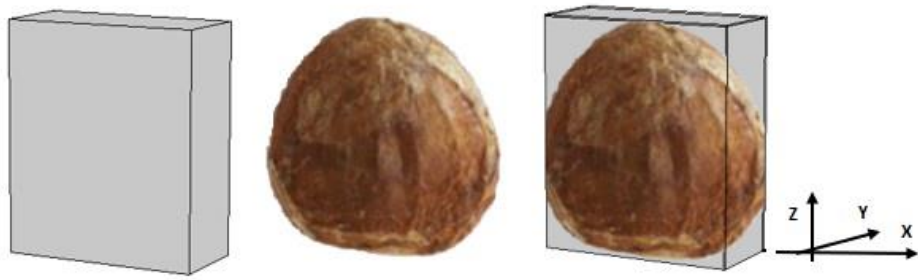

Fonte: LIMA (2018).

A Tabela 2 ilustra uma aproximação das dimensões da amêndoa do cupuaçu, que foram consideradas para a resolução das equações e simulação deste problema.

Tabela 2 - Dimensões da amêndoa do cupuaçu

$\begin{array}{cc}(X, Y, Z) & \text { Dimensões da amêndoa no cupuaçu } \\ \text { a } & 26 \mathrm{~mm} \\ \text { b } & 22 \mathrm{~mm} \\ \text { c } & 10 \mathrm{~mm}\end{array}$

No sistema de coordenadas retangulares $(x, y, z)$, a técnica de separação de variáveis para equação (7) é: 


$$
\psi(x, y, z)=X(x) Y(y) Z(z)(\mathbf{8})
$$

A equação (7) é desacoplada mediante a constante $-\lambda^{2}$ e pode ser escrita no sistema de coordenadas retangulares como:

$$
\frac{\nabla^{2} \psi(x, y, z)}{\psi(x, y, z)}=-\lambda^{2}
$$

Sendo que para cada sistema de coordenada geométrica o operador laplaciano $\nabla^{2} \psi$ tem uma forma diferente, para o caso investigado, coordenadas retangulares, o operador laplaciano correspondente é:

$$
\nabla^{2} \psi=\frac{\partial^{2} \psi}{\partial x^{2}}+\frac{\partial^{2} \psi}{\partial y^{2}}+\frac{\partial^{2} \psi}{\partial z^{2}}
$$

Usando na Eq. (7) com o respectivo operador laplaciano, tem se,

$$
\frac{1}{X} \frac{\partial^{2} X}{\partial x^{2}}+\frac{1}{Y} \frac{\partial^{2} Y}{\partial y^{2}}+\frac{1}{Z} \frac{\partial^{2} Z}{\partial z^{2}}+\lambda^{2}=0 \rightarrow \frac{X^{\prime \prime}}{X}+\frac{Y^{\prime \prime}}{Y}+\frac{Z^{\prime \prime}}{Z}+\lambda^{2}=0
$$

A partir da equação (10), é possível determinar cada uma das funções $(X, Y, Z)$. Que corresponde a:

$$
\begin{gathered}
X(x)=A \cos \left(k_{x} x\right)+B \operatorname{sen}\left(k_{x} x\right) \\
Y(y)=C \cos \left(k_{y} y\right)+D \operatorname{sen}\left(k_{y} y\right) \\
Z(z)=E \cos \left(k_{z} z\right)+F \operatorname{sen}\left(k_{z} z\right)
\end{gathered}
$$

onde $A, B, C, D, E$ e $F$ são constantes.

Com vinculo dado por

$$
k_{x}^{2}+k_{y}^{2}+k_{z}^{2}=\lambda^{2}
$$

Os resultados de (11) são substituídos em (8) e (3), chega se:

$$
\begin{aligned}
\Psi(x, y, z, t)=\{ & \left.A \cos \left(k_{x} x\right)+B \operatorname{sen}\left(k_{x} x\right)\right\}\left\{C \cos \left(k_{y} y\right)+D \operatorname{sen}\left(k_{y} y\right)\right\} \times \\
& \left\{E \cos \left(k_{z} z\right)+F \operatorname{sen}\left(k_{z} z\right)\right\} G e^{-\alpha \lambda^{2} t} \text { (13) }
\end{aligned}
$$

Vamos impor que as condições de fronteira no prisma retangular na Figura 3, tenham nas faces temperaturas nulas dadas por:

$$
\begin{gathered}
\Psi(0, y, z, t)=\Psi(a, y, z, t)=0 ; \Psi(x, 0, z, t)=\Psi(x, b, z, t)=0 ; \\
\Psi(x, y, 0, t)=\Psi(x, y, c, t)=0
\end{gathered}
$$

e a condição inicial, tempo igual a zero, temos uma temperatura $T_{0}$ ou seja

$$
\Psi(x, y, z, 0)=T_{o}
$$

Usando as condições dadas anteriormente em (13), resulta na temperatura:

$$
\begin{gathered}
\Psi(x, y, z, t)=\frac{64 T_{0}}{\pi^{3}} \sum_{n=0}^{+\infty} \sum_{m=0}^{+\infty} \sum_{p=0}^{+\infty} \frac{1}{(2 n+1)(2 m+1)(2 p+1)} \times \\
\operatorname{sen}\left(\frac{2 n+1}{a} \pi x\right) \operatorname{sen}\left(\frac{2 m+1}{b} \pi y\right) \operatorname{sen}\left(\frac{2 p+1}{c} \pi z\right) \times \\
e^{-\alpha \pi^{2}\left[\left(\frac{2 n+1}{a}\right)^{2}+\left(\frac{2 m+1}{b}\right)^{2}+\left(\frac{2 p+1}{c}\right)^{2}\right] t}
\end{gathered}
$$

onde $a, b$ e $c$ as dimensões da amêndoa investigada Tabela $2,(x, y, z)$ os pontos internos e $\mathrm{T}_{0}=70^{\circ} \mathrm{C}$ é a temperatura inicial dentro da amêndoa. 
Estas condições foram testadas no software Mathematica ${ }^{\circledR}$, e o resultado é apresentado na Figura 4.

Figura 4 - Condições iniciais e de fronteira

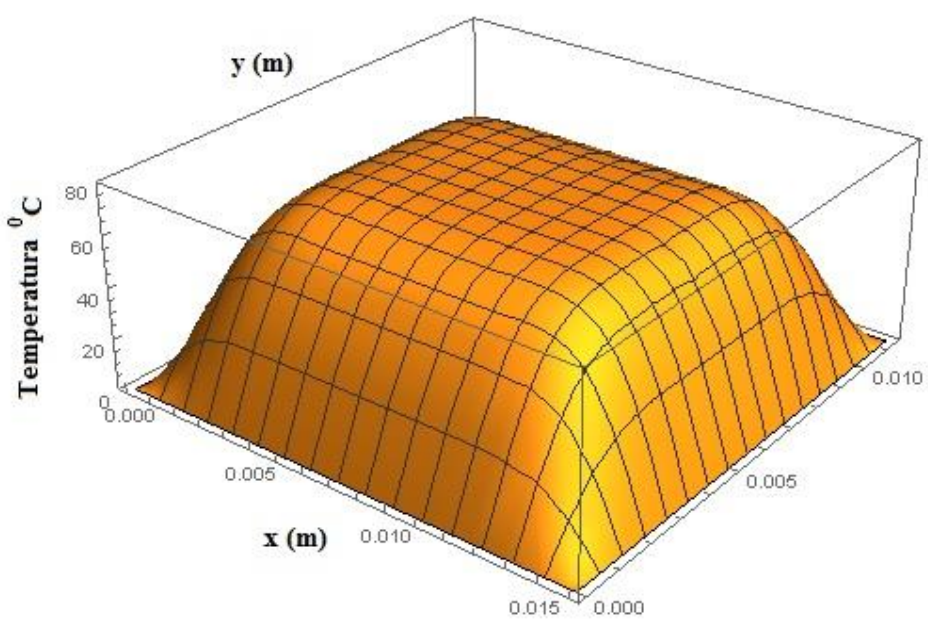

Fonte: LIMA (2018).

A Figura 4 mostra que nos extremos do modelo da amêndoa, nas faces, a temperatura é nula. 0 teste é fundamental para verificar se a solução 14 respeita as condições de contorno e inicial.

\section{MODELAGEM COMPUTACIONAL}

Nesta seção a modelagem tem as condições de contorno semelhante ao caso da seção anterior, porém com a temperatura na superfície da amêndoa a $25^{\circ} \mathrm{C}$ em equilíbrio a temperatura ambiente. No Anexo está o passo a passo para modelagem deste trabalho.

A amêndoa do cupuaçu é representada por meio do modelo prismático. Para a construção da geometria prismática, foi utilizada a ferramenta disponível no COMSOL "geometria" usando a opção "bloco". Com largura de $26 \mathrm{~mm}$, espessura de $22 \mathrm{~mm}$ e altura de $10 \mathrm{~mm}$, Tabela 2 . A Figura 5 ilustra o prismático para a representação da amêndoa do cupuaçu.

Figura 5 - Representação do modelo prismático

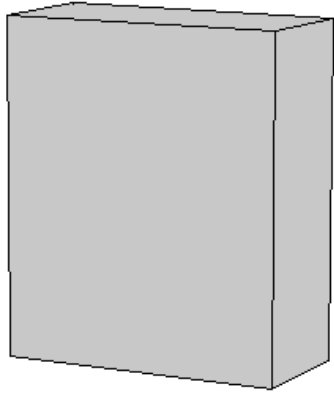

Fonte: LIMA (2018). 
Após desenhada a geometria, deve-se informar ao software quais as características do material que será simulado. COMSOL não possui em sua biblioteca o material equivalente ao que compõe o cupuaçu, por isso foi necessário criar um novo material com as seguintes propriedades térmicas: calor específico $\rho=3,69 \mathrm{KJkg}^{-1} \mathrm{~K}^{-1}$, massa específica $c=964,44 \mathrm{Kgm}^{-3}$ e condutividade térmica $k=0,135 \mathrm{KWm}^{-1} \mathrm{~K}^{-1}$. Com isso a difusividade é obtida por $\alpha=\frac{k}{\rho c}=$ $37,93 \times 10^{-6} \mathrm{~m}^{2} / \mathrm{s}$ (LIMA, 2018).

O domínio foi discretizado usando uma malha tetraédrica, tipo fina. A malha de cálculo subdivide o modelo em infinitesimais partes, permitindo que as equações diferenciais parciais sejam solucionadas por meio dos elementos finitos. Quanto mais refinada é a malha mais precisos são os cálculos (SOGLIA, 2019), porém maior é o esforço computacional, como um prisma é uma figura geométrica simples, foi utilizada a malha fina para obter cálculos com boa precisão. A Figura 6 apresenta a malha criada para a geometria prismática.

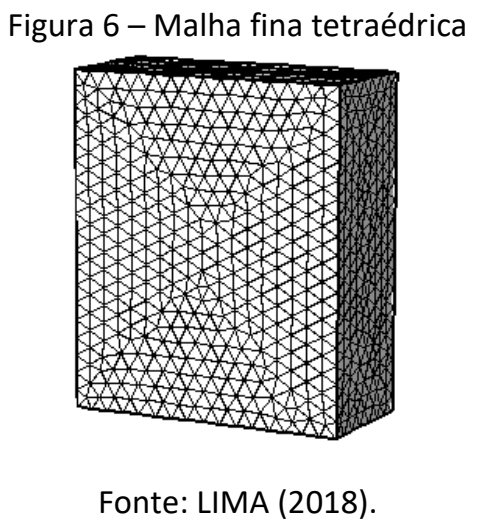

A Tabela 3 apresenta as características da malha gerada ilustrada na Figura 6.

Tabela 3 - Número e tipos de elementos para discretização do modelo prismático

\begin{tabular}{cc} 
Tipos de elementos & Número de elementos \\
Tetraédrico & 31.778 \\
Triangular & 2.682 \\
Aresta & 168 \\
Vértice & 8 \\
\hline
\end{tabular}

Criada a malhada de cálculo, é possível simular o sistema. A simulação consistiu na avaliação no comportamento de resfriamento da amêndoa do cupuaçu, com temperatura inicial de $70^{\circ} \mathrm{C}$, a amêndoa sofreu uma redução da temperatura devido a dissipação do calor para o ambiente, com temperatura ambiente de $25^{\circ} \mathrm{C}$.

A Figura 7 apresenta o comportamento de resfriamento do modelo prismático para os tempos de simulação de $0 \mathrm{~min}, 2,5 \mathrm{~min}, 12,5 \mathrm{~min}, 20 \mathrm{~min}, 40 \mathrm{~min}, 60 \mathrm{~min}$. Em que se observa que o calor vai sendo dissipado pelo ambiente, fazendo com o que a temperatura, do volume total, do modelo prismático, que inicialmente apresentava temperatura de $70^{\circ} \mathrm{C}$, tenha o calor dissipado para o ambiente com 
temperatura externa de $25^{\circ} \mathrm{C}$. Desta forma, verifica-se o comportamento em função do tempo tende ao equilíbrio térmico. Satisfazendo a primeira e segunda lei da termodinâmica.

Figura 7 - Simulação de resfriamento do modelo prismático durante 60 min
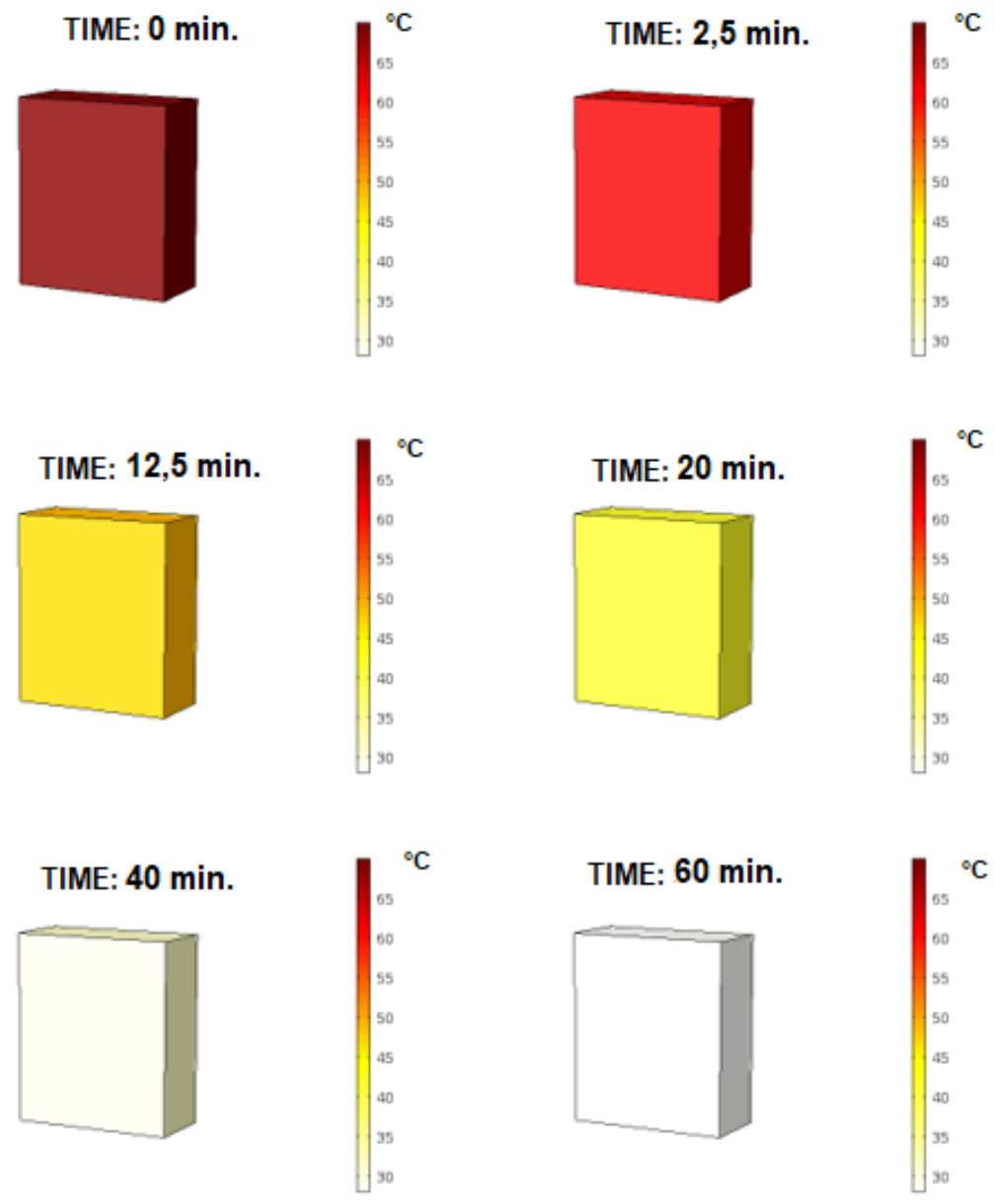

Fonte: LIMA (2018).

A Figura 7 revela que há uma redução na temperatura do modelo prismático em função do tempo, devido a dissipação do calor contido no seu interior para o ambiente, com temperatura de $25^{\circ} \mathrm{C}$.

A Figura 8 apresenta a curva de resfriamento da amêndoa do cupuaçu em função do tempo. 
Figura 8 - Resfriamento do modelo prismático, em função do tempo

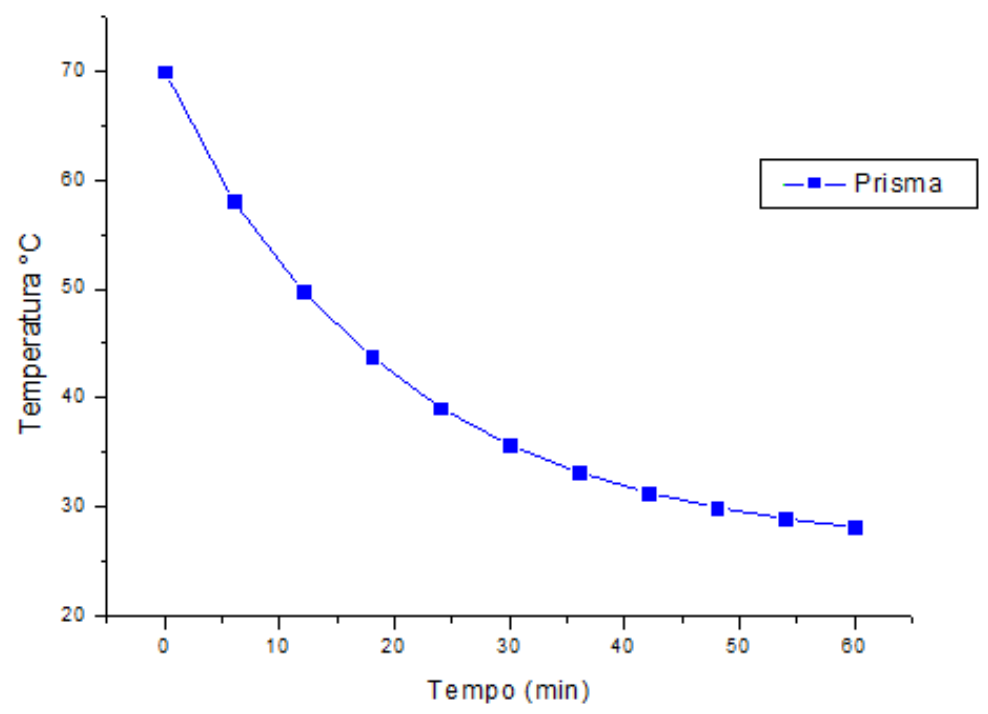

Fonte: LIMA (2018).

Observando a Figura 8, verifica-se que a redução na temperatura segue uma curva (curva exponencial) nos primeiros minutos de simulação, assumindo um comportamento mais atenuado a partir de 30 minutos.

Na Figura 3 é diferente da Figura 8, pois a amêndoa tem temperatura zero na superfície e temperatura inicial de $70^{\circ} \mathrm{C}$. Na Figura 8 a amêndoa tem temperatura inicial $70^{\circ} \mathrm{C}$ e na superfície de $25^{\circ} \mathrm{C}$ que é igual a temperatura ambiente.

\section{CONCLUSÃO}

Em virtude do exposto, verifica-se que os modelos computacionais podem ser aplicados no ensino de Ciência e Tecnologia. Neste trabalho foi descrita a curva de resfriamento da amêndoa do cupuaçu por meio da resolução da equação de Fourier em coordenadas cartesianas. A partir da resolução analítica, o modelo foi simulado computacionalmente e obtido a curva de resfriamento da amêndoa do cupuaçu. É observado na Figura 8 que há uma redução da temperatura de maneira mais intensa nos primeiros minutos de simulação e após 30 minutos a curva apresenta um comportamento mais tênue, tendendo à temperatura ambiente, que é de $25^{\circ} \mathrm{C}$. Esta metodologia pode ser replicada em diferentes sistemas, possibilitando conhecer o comportamento de diversos modelos. Desta forma, como sugestão de trabalho futuro, propõe-se avaliar a curva de resfriamento da amêndoa do cacau, utilizando um modelo de coordenadas prismática. 


\title{
DIDACTIC PROCEDURE FOR COMPUTATIONAL MODELING ON CUPUAÇU ALMOND IN CARTESIAN COORDINATES
}

\begin{abstract}
This article proposes an example of didactics aimed at the graduate and postgraduate students for modeling materials using a Cartesian coordinate system. The investigated material was the cupuaçu almond, with the objective of describing the cooling curve of the almond. For this, the methodology used consisted of the analytical resolution of the Fourier equation in Cartesian coordinates and computational model simulation. The simulation evaluated the heat dissipation of the almond, which had an initial temperature of $70^{\circ} \mathrm{C}$, and was submitted to an ambient temperature of $25^{\circ} \mathrm{C}$. The cupuaçu almond cooling curve reveals a rapid reduction in temperature in the first minutes of simulation, assuming a slower behavior after $30 \mathrm{~min}$.
\end{abstract}

KEYWORDS: Heat transfer. Fick's equation. Teaching. 


\section{AGRADECIMENTOS}

Sales agradece ao CNPq 315519/2018-5, CAPES e FAPESB - PIE 0013/2016 pelo apoio e Lima a FAPESB processo 0048/2017 pelo apoio.

\section{REFERÊNCIAS}

ARFKEN, G. B.; WERBER, H. J. Métodos Matemáticos para Engenharia e Física. 6. ed. Rio de Janeiro: Elsevier Brasil, 2007.

COHEN, K. O.; LUCCAS, V.; SOUSA, M. V.; JACKIX, M. N. H. Processamento Tecnológico das Amêndoas de Cacau e Cupuaçu. Belém: Embrapa Amazônia Orienta, 2003.

COMSOL. COMSOL Multiphysics. 2017. Accessed: 2017-19-07.

CHWIF, L.; MEDINA, A. C. Modelagem e Simulação de Eventos Discretos: Teoria e Aplicações. 4. ed. São Paulo: Elsevier Brasil, 2014.

EMBRAPA. Empresa Brasileira de Pesquisa Agropecuária Ministério da Agricultura, Pecuária e Abastecimento. Embrapa Amapá: Multimídia: Banco de Imagens, 2016.

LIMA, G. P. A. Modelando a Difusividade Térmica da Amêndoa do Cupuaçu. Dissertação (Mestrado em Modelagem Computacional em Ciência e Tecnologia) Universidade Estadual de Santa Cruz, Ilhéus, 2018.

LIMA, G. P. A.; SALES, J. H.; PORTUGAL, A. A. Sistema alternativo para secagem de cacau e geração de energia. Revista GEINTEC: Gestão, Inovação e Tecnologias, v.5, n.1, p.1703-1715, 2015.

LIMA, G. P. A.; SALES, J. H. Difusividade térmica da amêndoa do cupuaçu. XX ENMC - National Meeting on Computational Modeling and VIII ECTM - Meeting on Materials Science and Technology. Nova Friburgo, RJ: Anais, 2017.

LOTT, R. S.; MACHADO, A.; MAZZIEIRO N. T. ANDE JÚNIOR, J. L. Aplicabilidade científica do método dos elementos finitos. Revista Dental Press de Ortodontia e Ortopedia Facial, v. 11, n. 2, p. 35-43, 2006.

MCGUINNESS, N.; WILSON, A. N.; JONES, M.; MIDDLETON, J.; ROBERTSON, N. R. Stresses induced by edgewise appliances in the periodontal ligament - a finit 
NAZARÉ, R. F. R.; BARBOSA, W. C.; VIÉGAS, R. M. F. Processamento das sementes de cupuaçu para a obtenção de cupulate. Belém, 1990. 38 p.

SALES, J. H.; CÂNDIDA, T. Efeito da temperatura sobre a amêndoa de cacau: Secador Vertical. Revista GEINTEC: Gestão Inovação e Tecnologia, v. 6, n. 3, p. 3437-3446, 2016.

SALES, J. H.; LIMA, G. P. A. Difusividade térmica do cacau em função da geometria do modelo. Revista SODEBRAS, v. 13, n. 149, p. 31-34, 2018.

SASSERON, J. L. Avaliação de propriedades físicas e curvas de secagem, em camadas finas, de amêndoas de cacau (Theobromacacao L.). Dissertação (Mestrado) - Dissertação (Mestrado em Engenharia Agrícola) - Universidade Federal de Viçosa, 1984.

SOGLIA, L. V. M. Sistema Auxiliar para Secagem de Grãos. Dissertação (Mestrado em Modelagem Computacional em Ciência e Tecnologia) - Universidade Estadual de Santa Cruz, Ilhéus, 2019.

SOUZA, A. G. C.; SILVA, S. E. L.; TAVARES, A. M.; RODRIGUES, M. R. L. A cultura do cupuaçu (theobroma grandflorum (willd. ex spreng.) schum.)). Embrapa Amazônia Ocidental Manaus, v. 1, p. 39, 1999.

YANG, H.; PROTIVA, P.; CUI, B.; MA, C.; BGGETT, S.; HEQUET, V.; MORI, S.; WEINSTEIN, I.; KENNELLY, E. New bioactive polyphenols from theobrama grandiflorum ("cupuaçu"). Journal of Natural Products., v. 66, p. 1501-1504, 2003. 


\section{ANEXOS}

\section{ANEXO. SIMULAÇÃO COMPUTACIONAL}

Para simular o comportamento térmico foi utilizado software COMSOL, a sequência de passos empregados na simulação, ilustrada na Figura 2 é detalhada neste apêndice. A Figura 9 apresenta o modo de simulação escolhido, que foi o "model wizard". E este consiste em um modo inteligente, presente no software, que facilita a construção do modelo que será simulado.

Figura 9 - Escolha do modelo: Model wizard

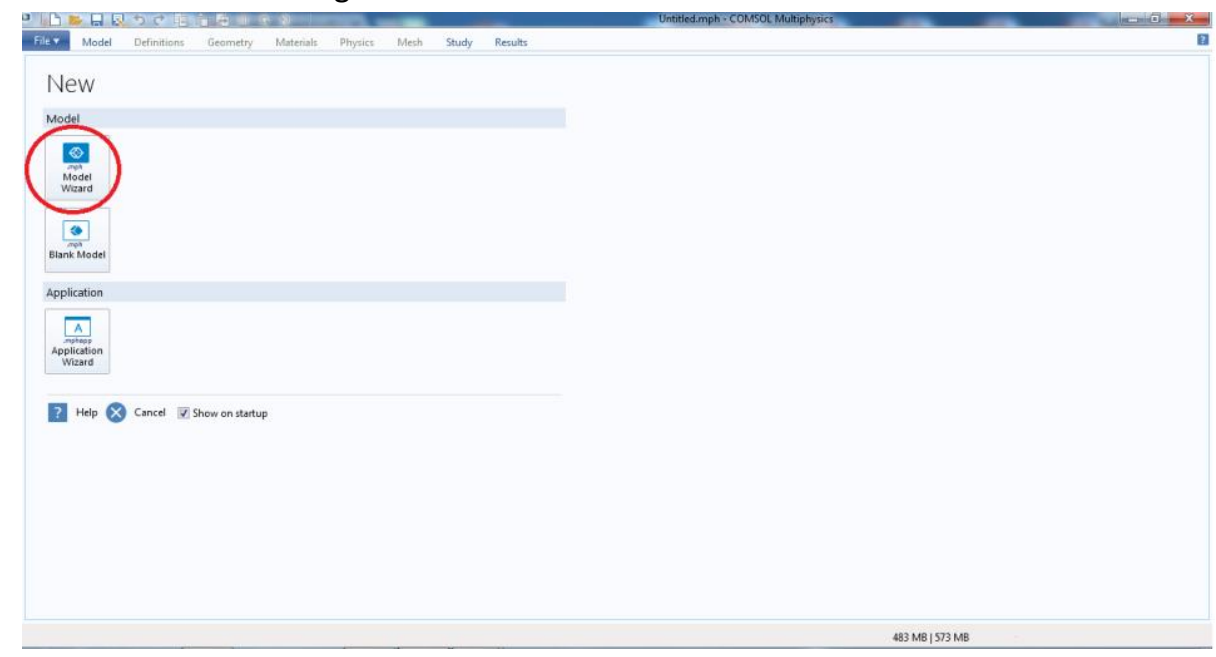

Fonte: LIMA (2018).

Após a escolha do modo de simulação, deve ser selecionado a dimensão do espaço que se deseja operar. A Figura 10 apresenta a dimensão escolhida do espaço trabalhado, que foi a tridimensional. Para facilitar a observação do comportamento da dissipação do calor no interior de cada geometria, sendo mais conveniente trabalhar em três dimensões.

Figura 10 - Escolha da dimensão: 3D

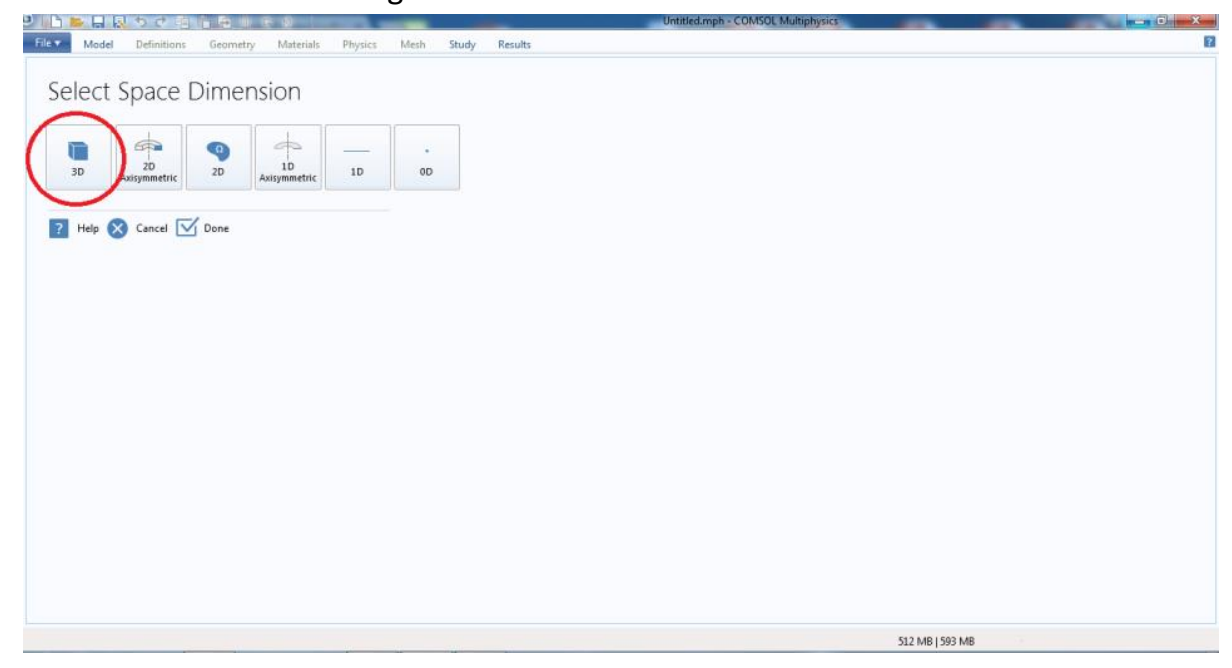


Para o estudo de mecanismos de transferência de calor pelo COMSOL, é necessário determinar os métodos físicos que compreende o estudo. A Figura 11 mostra o método físico utilizado na solução do problema de transferência de calor, que consistiu na "Heat transfer module", módulo de transferência de calor, o qual possibilita o estudo de situações onde há geração, absorção ou transferência de calor ou energia.

Figura 11 - Escolha do método físico: Heat transfer module

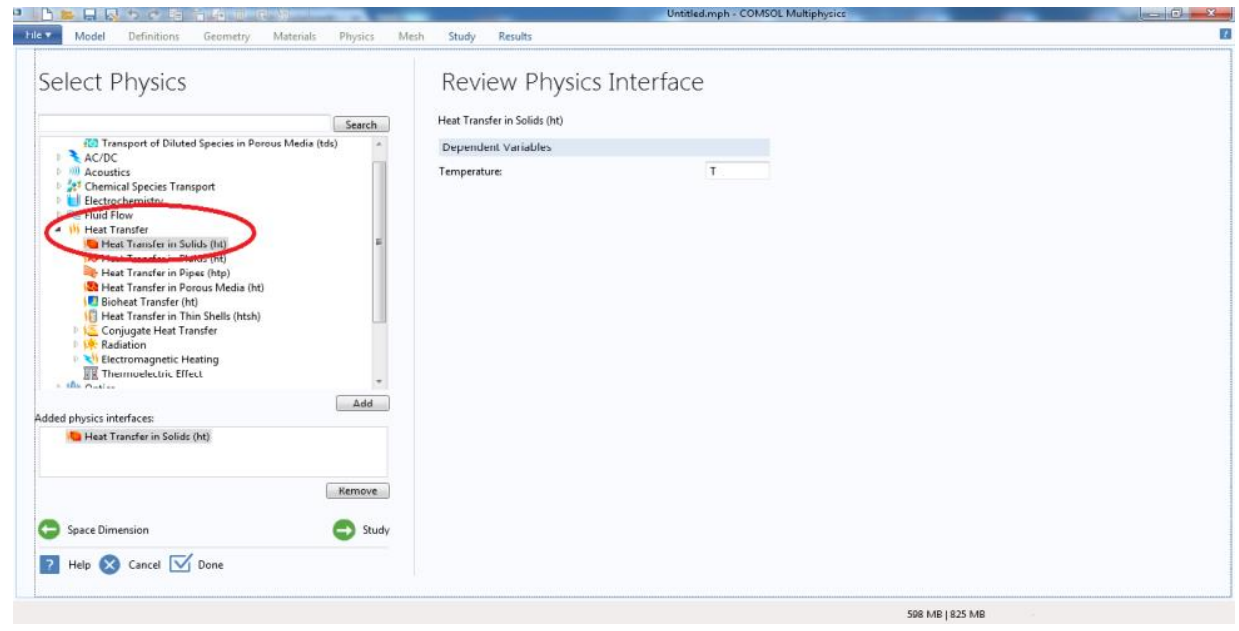

Fonte: LIMA (2018).

O comportamento do resfriamento da amêndoa do cupuaçu não ocorre em regime estacionário, ele é dependente do tempo, desta forma o tipo de estudo escolhido foi, "Time dependent", que depende do tempo, ilustrado na Figura 12.

Figura 12 - Tipo de estudo: Time dependente

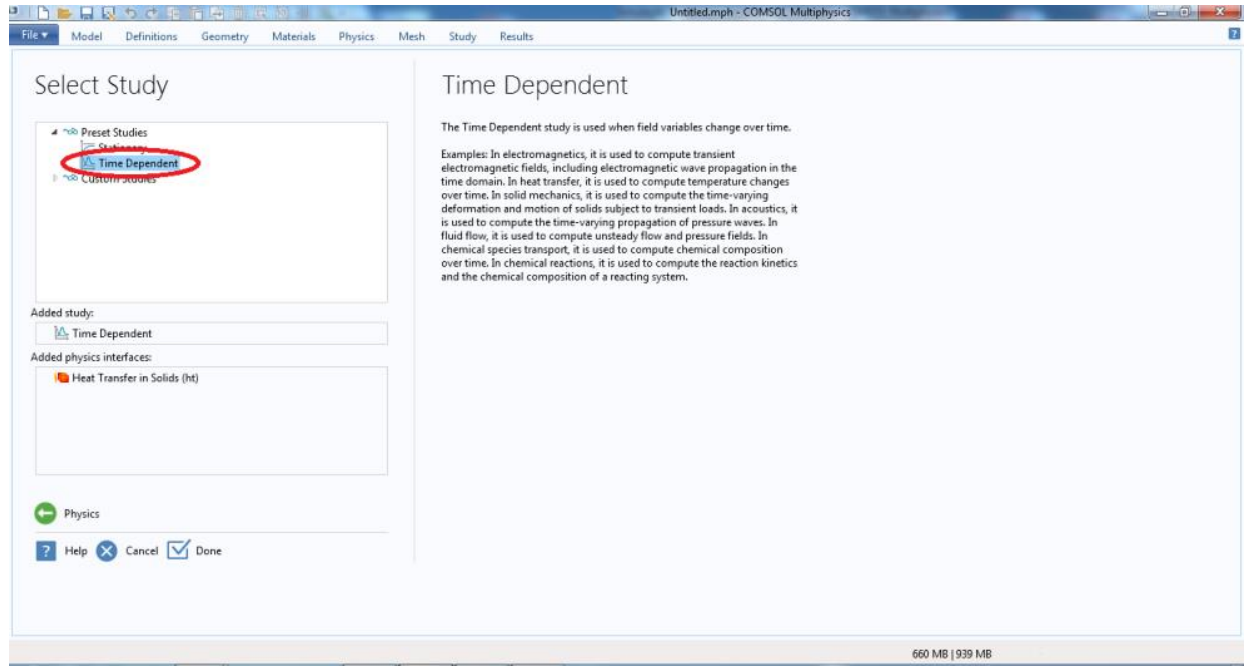

Fonte: LIMA (2018).

Como na biblioteca do COMSOL, não possui material equivalente ao cupuaçu, foi necessário criar um material com as propriedades termofísicas. Além disso, as dimensões para criar o modelo foi equivalente as dimensões médias da amêndoa do cupuaçu. Desta forma, os parâmetros usados na simulação são ilustrados na Figura 13. 
Figura 13 - Parâmetros da simulação

\begin{tabular}{|l|l|l|l|}
\hline "Name & Expression & Value & Description \\
\hline a & $26[\mathrm{~mm}]$ & $0.026 \mathrm{~m}$ & Coordenada x (valor maior) \\
\hline b & $22[\mathrm{~mm}]$ & $0.022 \mathrm{~m}$ & Cordenada y (valor intermediário) \\
\hline c & $10[\mathrm{~mm}]$ & $0.01 \mathrm{~m}$ & Coordenada z (valor menor) \\
\hline T_amendoa & $70[\mathrm{degC}]$ & $343.15 \mathrm{~K}$ & Temperatura inicial na amêndoa \\
\hline ccu & $3.69\left[\mathrm{~kJ} /\left(\mathrm{kg}^{\star} \mathrm{K}\right)\right]$ & $3690 \mathrm{~J} /(\mathrm{kg} \cdot \mathrm{K})$ & Calor específico \\
\hline pcu & $964.44\left[\mathrm{~kg} / \mathrm{m}^{\wedge} 3\right]$ & $964.44 \mathrm{~kg} / \mathrm{m}^{3}$ & Massa específica \\
\hline $\mathrm{kcu}$ & $0.135\left[\mathrm{~kW} /\left(\mathrm{m}^{\star} \mathrm{K}\right)\right]$ & $135 \mathrm{~W} /(\mathrm{m} \cdot \mathrm{K})$ & Condutividade térmica \\
\hline
\end{tabular}

Fonte: LIMA (2018).

Recebido: 11 jul. 2018.

Aprovado: 30 jul. 2020.

DOI: $10.3895 /$ rbect.v13n3.8517

Como citar: SALES, J. H. O.; LIMA, G. P. A. Procedimento didático para a modelagem computacional da amêndoa do cupuaçu em coordenadas cartesianas. Revista Brasileira de Ensino de Ciência e

Tecnologia, Ponta Grossa, v.13, n. 3, p. 1-19, set./dez. 2020. Disponível em:

$<$ https://periodicos.utfpr.edu.br/rbect/article/view/8517>. Acesso em: XXX

Correspondência: Jorge Henrique de Oliveira Sales - jhosales@uesc.br

Direito autoral: Este artigo está licenciado sob os termos da Licença Creative Commons-Atribuição 4.0 Internacional. 